IP Periodica Polytechnica Mechanical Engineering

\author{
62(1), pp. 42-50, 2018 \\ https://doi.org/10.3311/PPme.11382 \\ Creative Commons Attribution (i)
}

RESEARCH ARTICLE

\section{The Effects of Aiding and Opposing Thermal Buoyancy on Downward Flow around a Confined Circular Cylinder}

\author{
Houssem Laidoudi ${ }^{1 *}$, Mohamed Bouzit ${ }^{1}$
}

Received 12 August 2017; accepted 25 September 2017

\begin{abstract}
In this paper, two-dimensional simulations are carried out to understand the effects of aiding and opposing thermal buoyancy on the downward flow and heat transfer characteristics around a heated/cold circular cylinder confined in vertical channel. The numerical results have been presented and discussed for the range of conditions as: $R e=5$ to $40, R i=-1$ to 1 at fixed $\operatorname{Pr}=1$ and blockage ratio $\beta=0.2$. The steady simulations are performed with a finite volume method based on ANSYS-CFX code. The main results are presented in terms of streamlines and isotherm contours to interpret the obtained phenomena. In addition, the average Nusselt number is computed to determine the role of Reynolds and Richardson numbers on heat transfer rate. It is found that increase in the effect of aiding buoyancy reduces the heat transfer rate but increase in the effect of opposing buoyancy enhances the heat transfer rate. Moreover, for aiding buoyancy condition creates some counter rotating regions in upstream region.
\end{abstract}

\section{Keywords}

heat transfer, Nusselt number, Richardson number, steady flow, mixed convection

\footnotetext{
${ }^{1}$ Laboratory of Sciences and Marine Engineering, Faculty of Mechanical Engineering, USTO-MB, BP 1505, El-Menaouer, Oran, 13000, Algeria

*Corresponding author, e-mail: houssem.laidoudi@univ-usto.dz
}

\section{Introduction}

The understanding of mixed convection from confined bluff bodies is the key issue in design and development of such products. In recent years, significant research efforts have been conducted in studying the momentum and heat transfer characteristics from a long circular cylinder immersed in fluids. This typical cylinder which can be used in different applications such as in novel heat exchangers, cooling electronic components, nuclear system, in polymer engineering applications, in continuous thermal treatment of food stuffs, etc.

Principally the mixed convection occurs when natural and forced convection mechanisms act simultaneously to transfer heat. This is also known as situations where both pressure and buoyant forces interact. The single parameter that determines the relative interaction of natural convection and forced convection is the Richardson number, defined as, $R i=G r / R e^{2}$ with $G r$ and $R e$ being the Grashof and Reynolds numbers. For $R i>$ 1 , the natural convection dominates over the forced convection and for $R i<1$ the forced convection dominates. Both the natural and forced convection are equally important when $R i$ is close to 1 . For the downward flow and based on literature of [2] and [8], the effect of thermal buoyancy on fluid flow can be divided into two parts, the first when the cylinder is heated and there is motion of fluid particles around the cylinder towards the upward direction caused by the force of opposing buoyancy. And the second when the cylinder is cooled and the fluid particles around the cylinder induced by the force of aiding buoyancy move with same direction of fluid flow (downward direction). Theoretically, the positive value of Richardson number hints that the buoyancy effect is opposed. Meanwhile, the negative value determines that the buoyancy is superimposed.

For incompressible Poiseuille fluids, there are some thorough reviews on the effects of aiding and opposing buoyancy characteristics on fluid flow and heat transfer from vertical circular [1-3] square [4-7], semi-circular [8] and triangular [9]. Laidoudi and Bouzit [1] studied numerically the influence of aiding buoyancy on confined upward flow of power-law fluids around heated cylinder within a vertical adiabatic channel. The work emphasized mainly on the influence of aiding thermal buoyancy 
strength and velocity of the flow on recirculation region behind the obstacle and heat transfer rate. The detailed flow and temperature fields are visualized in term of streamlines and isotherm contours. The numerical results have been presented and discussed for the range of conditions as $10 \leq R e \leq 40$, Richardson number $0 \leq R i \leq 1$ at Prandtl number $\operatorname{Pr}=1$, and blockage ratio $\beta=0.2$. It is found that increase in the strength of buoyancy diminishes gradually the flow separation behind the obstacle. Salcedo et al. [2] presented a two dimensional numerical investigation of laminar downward flow around a pair of equal-sized isothermal cylinders in tandem arrangement confined in vertical channel under the effect of aiding and opposing buoyancy. The governing equations are solved in unsteady regime for the range of these conditions as: $R i=-1$ to 4 , the longitudinal spacing ratio $\sigma=1$ to 5 , at $\operatorname{Re}=200, \operatorname{Pr}=7$ and blockage ratio $\beta=0.2$. The obtained results showed how the previous parameters affect wake structure and vortex shedding. Also, the local and average Nusselt numbers of both cylinders are presented. Chatterjee and Mondal [3] analyzed through a numerical simulation the phenomena of suppression of flow separation behind bluff obstacles (circular and square) under aiding thermal buoyancy. For that purpose, they consider an unconfined flow of a Newtonian fluid around heated cylinders of circular and square cross-sections in a buoyancy-aided vertical upward flow configuration. The research is undertaken in the flowing ranges: $R e=10$ to 40, $R i=0$ to 1 and $P r=0.7$ to 50. It is observed that the square cylinder requires more heating in comparison to its circular counterpart of equal linear dimension for complete suppression of flow separation. Furthermore, the effect of Prandtl number on suppression of flow separation is also investigated and it is found that the high Prandtl number fluid requires less heating for suppression. Sarkar et al. [4] numerically investigated the effect of aiding and opposing buoyancy for the values of Richardson number of ( $R i=-1$ to 1 ) and for Reynolds number fixed at $R e=100$ and $\operatorname{Pr}=0.7$ on upward flow around two square cylinders in vertical tandem arrangement, the governing equations are solved in unsteady regime in order to test the buoyancy strength on the vortex shedding behind the obstacle. It is found that for the aiding buoyancy condition, increase in Richardson number decreases the vortex shedding. Chatterjee and Raja [5] studied numerically the mixed convection of upward flow around five in-line isothermal square cylinders arranged within a vertical duct. In the transient regime, the covering equations that involve continuity, momentum, and energy equations are carried out by finite volume based commercial computational fluid dynamics package FLUENT, the initial conditions are limited in the following ranges: $R i=-1$ to $1, \beta=0 \backslash \%-50 \backslash \%$, and $\operatorname{Pr}=0.7, \operatorname{Re}=100$. The results showed that the average $N u$ is almost invariant with $R i$ at higher blockage, whereas a local minimal is observed close to $R i=0$ for unconfined case, and the average $N u$ increases with the blockage ratio. Sharma et al. [6] they carried out the mixed convection heat transfer around a square cylinder exposed in vertical flow under aiding thermal buoyancy, the cylinder is assumed to be unconfined the Reynolds and Richardson number are limited in these ranges $R e=$ 1 to $40, R i=1$ to 40 , The semi-explicit finite volume method implemented on the collocated grid arrangement is used to solve the governing equations, the results present that The friction, pressure and total drag coefficients are found to increase with Richardson number, that heat transfer increases with increase in Reynolds number and/or Richardson number. Sharma and Eswaran [7] studied the effect of channel confinement of various degrees and the effect of aiding/opposing thermal buoyancy on the upward fluid flow field and heat transfer from a confined heated/cold square cylinder, the initial conditions are: $R i=-1$ to $1, R e=100$, and $\operatorname{Pr}=0.7$. The results showed that with increasing blockage ratio, the minimum heating (critical $R i_{c}$ ) required for the suppression of vortex shedding decreases up to a certain blockage ratio $(\beta=30 \%)$. Also, Salcedo et al. [8] studied numerically the effect of aiding and opposing buoyancy on downward flow around confined semi-circular cylinders in tandem arrangement, the work studied principally the buoyancy strength and the distance between the obstacles for these conditions: $R i=-1$ to $1, \operatorname{Re}=200, \operatorname{Pr}=7$ and blockage ratio $\beta=0.2$. The streamlines and the isotherms are illustrated also the average Nusselt number is computed. The obtained results showed that how the previous parameters influence on the wake and vortex shedding behind the obstacles. Gupta et al. [9] numerically carried out the simulations of upward flow of Non-Newtonian power-law fluids around a confined triangular cylinder with two geometrical configurations under the effect of buoyancy. The governing equations are solved in laminar steady regime in the range of these conditions: $R e=1$ to 35 , power-law index $n=0.4$ to $1.8, R i=0$ to 2 at fixed value of Prandtl number of 50. It is found that when the buoyancy is superimposed the recirculation region behind the obstacle diminishes gradually.

Singh and Makinde [10] analyzed the flow for both assisting and opposing buoyancy and the effect of other parameters on the fluid velocity and temperature distribution which are presented graphically of viscous incompressible fluid along axial direction of axial direction of a vertical stationary isothermal cylinder.

For a horizontal channel, there have been some researches of the numerical investigation of fluid flow and mixed convection heat transfer characteristics around a confined cylinder with different cross-sectional form. Yahiaoui et al. [11] investigated the effect of thermal buoyancy of Newtonian fluid around a rotating circular cylinder. Chatterjee and Amiroudine [12] numerically investigated the effect of Richardson $R i=0$ to 1 and $P r=$ 0.7 to 100 on the flow and heat transfer from the tandem square cylinders in two-dimensional steady regime, for the $R e$ number range of 1 to 30 . It is found that the recirculation region behind the obstacle is found to increase with increase in Ri number the $N u$ number also increases with increase in $R i$ number. Sukesan et al. [13] studied the mixed convection heat transfer 


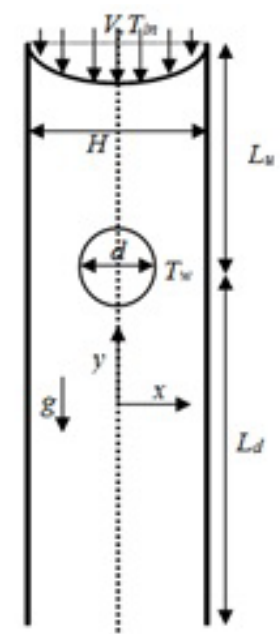

Fig. 1 Schematic diagram of the computational domain.

from confined semi-circular cylinder within a horizontal channel. The average Nusselt number and other flow characteristic parameter are presented to determine the effect of aiding thermal buoyancy. Laidoudi and Bouzit [14] simulated the incompressible Newtonian flow over a confined circular cylinder in horizontal channel under the effect thermal buoyancy.

Accordingly, the present work aims to extend the knowledge of the influences of thermal buoyancy by investigating numerically and simultaneously the effects of aiding and opposing-buoyancy mixed convective on downward flow and heat transfer characteristics of Newtonian fluid around a circular cylinder in a confined channel for the range of Reynolds numbers $R e=5$ to 40 , which characterizes a steady-flow regime, with a fixed value of Prandtl number $P r=1$ and blockage parameter of $\beta=0.2$ and a range of Richardson number $R i=-1$ to 1 . The objective is to analyze the influence of aiding and opposing thermal buoyancy and other physical parameters on the downward fluid dynamic and heat transfer characteristics.

\section{Physical problem and governing equations}

The problem under consideration is shown schematically in Fig. 1. A circular cylinder (of diameter $d$ ) heated or cooled to constant temperature $T_{w}$ is exposed to a developed downward velocity and constant temperature $T_{i n}$. In order to make the problem computationally feasible, artificial confining boundaries are placed around the flow where "no-slip" boundary condition is used for the two channel walls for the flow assumption and the channel walls are assumed to be adiabatic for the heat transfer assumption as it is shown in the Fig. 1. The ratio of cylinder diameter $(d)$ to the channel width $(H)$ defines the blockage ratio $(\beta=0.2)$. The upstream and downstream distances of the computational domain are selected as: $L_{u}=10 d, L_{d}=20 d$. We assume that the flow enters the channel under fully developed velocity profile and constant temperature $T_{i n}$.

The flow is assumed steady, two-dimensional, laminar and incompressible, for which the governing conservation dimensionless equations of mass, momentum, and energy with constant thermo-physical properties along with Boussinesq approximation and negligible dissipation effect can be written in the following forms:

- Continuity:

$$
\frac{\partial U}{\partial X}+\frac{\partial V}{\partial Y}=0
$$

- Momentum

$$
\begin{gathered}
U \frac{\partial U}{\partial X}+V \frac{\partial U}{\partial Y}=-\frac{\partial P}{\partial X}+\frac{1}{\operatorname{Re}}\left(\frac{\partial^{2} U}{\partial X^{2}}+\frac{\partial^{2} V}{\partial Y^{2}}\right) \\
U \frac{\partial V}{\partial X}+V \frac{\partial V}{\partial Y}=-\frac{\partial P}{\partial Y}+\frac{1}{\operatorname{Re}}\left(\frac{\partial^{2} U}{\partial X^{2}}+\frac{\partial^{2} V}{\partial Y^{2}}\right)+\operatorname{Ri\theta }
\end{gathered}
$$

- Thermal energy

$$
U \frac{\partial \theta}{\partial X}+V \frac{\partial \theta}{\partial Y}=\frac{1}{\operatorname{RePr}}\left(\frac{\partial^{2} \theta}{\partial X^{2}}+\frac{\partial^{2} \theta}{\partial Y^{2}}\right)
$$

In the above equations $U, V, P, \theta, R e, R i$ and $P r$ are dimensionless fluid velocities, temperature, pressure, Reynolds number, Richardson number, and Prandtl number $(\operatorname{Pr}=1)$, respectively. The dimensionless forms of the variables are:

$$
\begin{gathered}
U=\frac{u}{V_{\max }}, V=\frac{v}{V_{\max }}, X=\frac{x}{d}, Y=\frac{y}{d}, \\
P=\frac{p}{\rho V_{\max }^{2}}, \theta=\frac{T-T_{\text {in }}}{T_{w}-T_{i n}}, \\
R e=\frac{\rho d V_{\max }}{m}, R i=\frac{G r}{R e^{2}},
\end{gathered}
$$

where $G r$ is the Grashof number which can be written as:

$$
G r=\frac{g B\left(T_{w}-T_{i n}\right) d^{3} \rho^{2}}{\eta^{2}}
$$

where $g, B$ : is the gravitational acceleration, the volumetric expansion coefficient respectively.

The heat transfer between the cylinder and the surrounding fluid is calculated by the Nusselt number. The local Nusselt number based on the cylinder dimension is given by:

$$
N u=\frac{h d}{k}=-\frac{\partial \theta}{\partial n_{s}},
$$

where $h$ is the local heat transfer coefficient, $k$ is the thermal conductivity of the fluid and $n$ is the direction normal to the cylinder surface. Surface average heat transfer at the cylinder is obtained by integrating the local Nusselt number along the cylinder face.

The boundary conditions used the flow and heat configurations are:

$$
U=0, V=(1-|2 Y \beta|)^{(2)}, \theta=0
$$


At the inlet a fully developed velocity profile for laminar flow with a constant temperature, this is given by:

$$
U=0, V=(1-|2 Y \beta|)^{(2)}, \theta=0
$$

On the surface of the obstacle cylinder: The standard no-slip condition is used and the cylinder is maintained and heated with a constant temperature $T_{w}$

$$
U=0, V=0, \theta=1
$$

At the channel walls, the usual no-slip condition for flow and adiabatic condition for energy are used.

$$
U=0, V=0, \frac{\partial \theta}{\partial Y}=0
$$

At the outlet Neumann boundary condition for field variables is used:

$$
\frac{\partial U}{\partial X}=0, \frac{\partial V}{\partial Y}=0, \frac{\partial \theta}{\partial Y}=0
$$

\section{Solution methodology}

The numerical simulation is carried out by using the commercial CFD package ANSYS-CFX. This code which is developed by AEA Technology, UK, simulated the steady state 2D flow inside of our numerical domain under aiding and opposing buoyancy. The package uses a finite volume method, which first involves discretizing the spatial domain using a mesh. The mesh is used to construct finite volumes, which are used to conserve relevant quantities such as mass, momentum, and energy. The governing equations are integrated over each control volume, and Gauss' Divergence Theorem is applied to convert volume integrals involving divergence and gradient operators to surface integrals. The laminar viscous model is selected to account for the low Reynolds flow consideration. For more information about the package's schemas use the ANSYS user's guide [15]. The convergence criteria based on relative error for the inner iterations are set as $10^{-4}$ for the discretized continuity and momentum equations and $10^{-4}$ for the discretized energy equation, this value of error was selected based on validation test which is presented in our previous work [1].

Fig. 2 shows the typical grid applied for this study. This figure presents the unstructured trilateral cells of non-uniform grid points which are distributed in a non-uniform manner with higher concentration in the vicinity of the cylinder. Very small size control volume near the surface is adopted with a total number of cells 120358 elements. The mesh sizes as well as the upstream and downstream distances are adopted after performing a comprehensive grid independency study which presented in our earlier works [1] and [14].

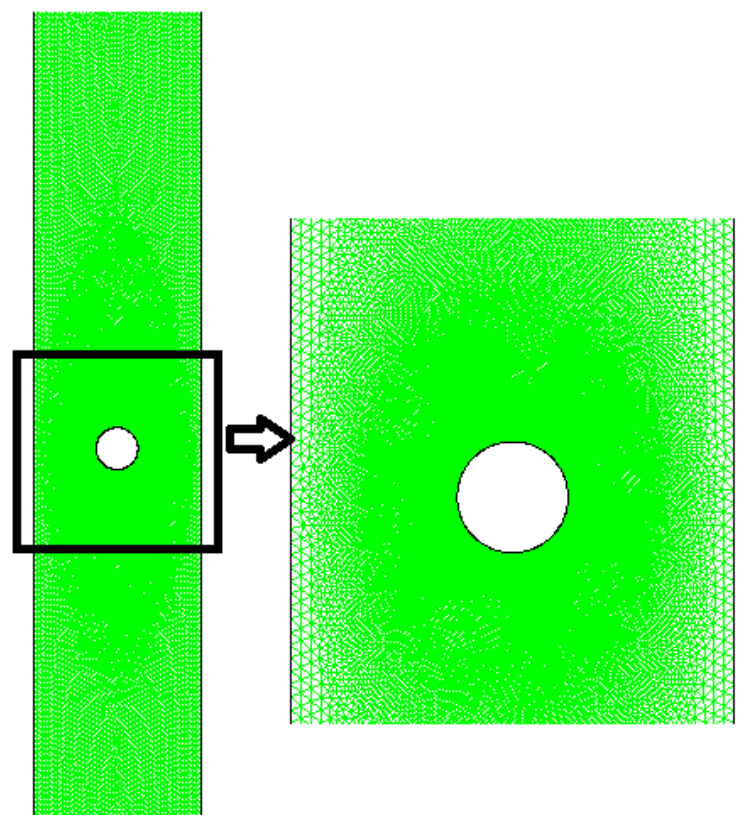

Fig. 2 Typical grids used for simulation.

\section{Results and discussion}

Numerical results are presented in term of streamlines and isotherm contours for the following range of parameters: $R e=$ 5 to 40 , and Richardson number, $R i=-1$ to 1 (positive values for the opposing and negative values for the aiding buoyancy case) at fixed values of $\operatorname{Pr}=1$ and $\beta=0.2$. The accuracy of the numerical simulation is validated and presented in our earlier works [1] and [14] for Nusselt number dependence on Reynolds number for various Richardson number and Prandtl number.

\section{Flow and isotherm pattern}

Figs. 3 and 4 show the streamline contours for $R e=10,20$ and 30 , respectively under the influence of opposing and aiding thermal buoyancy at $\beta=0.2$ and $\operatorname{Pr}=1$. The colors shown on streamlines indicate the flow velocity. The red coloration represents maximum velocity, while blue color indicates negative or counter rotating flow. For all cases, the flow is found to be symmetrical in nature with respect to the line $y=0$ for the range of Reynolds number considered. The streamlines contours show a closed recirculation region involving of twin symmetric vortices created behind the cylinder. For the case of opposing and aiding buoyancy, the size of the recirculation region both along the stream-wise as well as transversal directions increases with increase in the value of Reynolds number. Interesting variations of the size of recirculation region behind the cylinder are observed with respect to the Richardson number. The size of recirculation region behind the object decreases with increased cooling (decreasing $R i<0$ ) of the cylinder and increases with increased heating (increasing $R i>0$ ). 
(a) $R e=10$

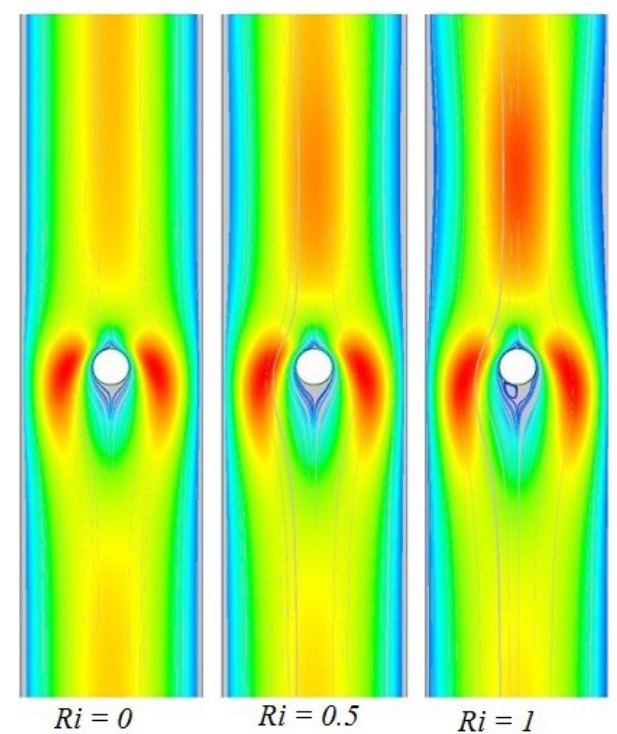

(b) $R e=20$
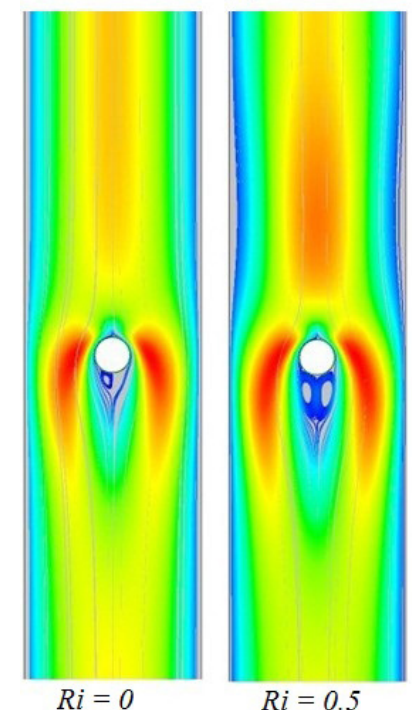

$R i=0.5$

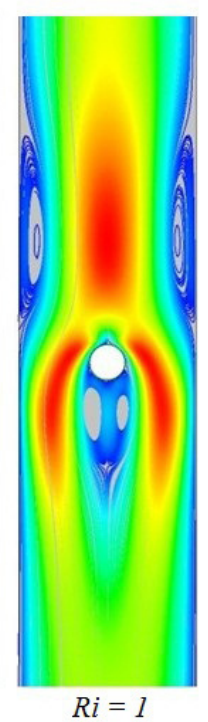

(c) $\operatorname{Re}=30$

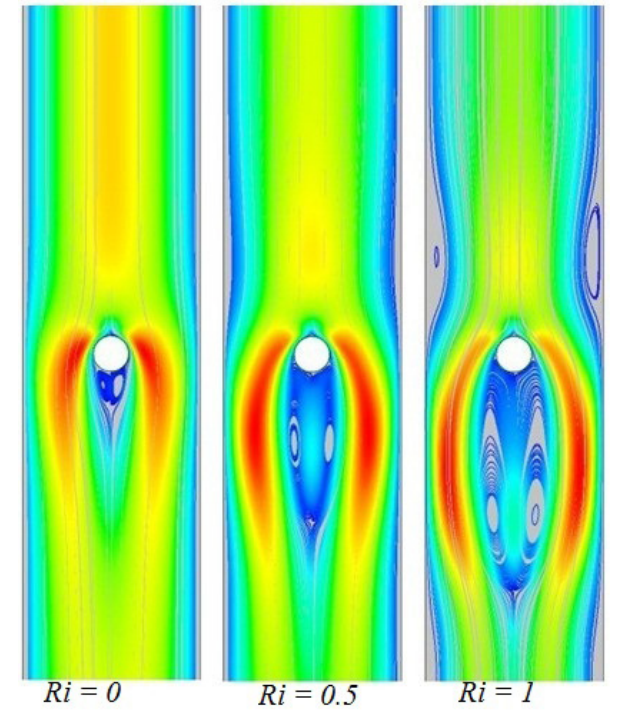

Fig. 3 Streamlines for different $R i$ and $R e$, under the influence of opposing buoyancy. (a) $\operatorname{Re}=10$

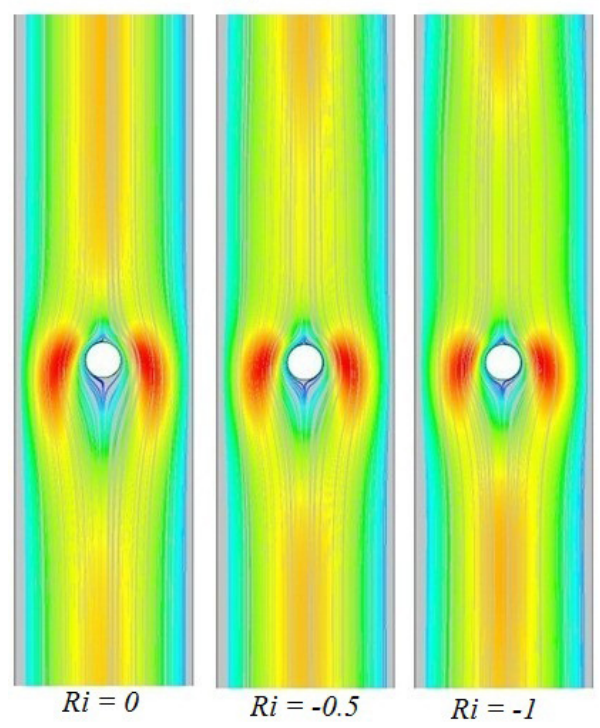

(b) $R e=20$

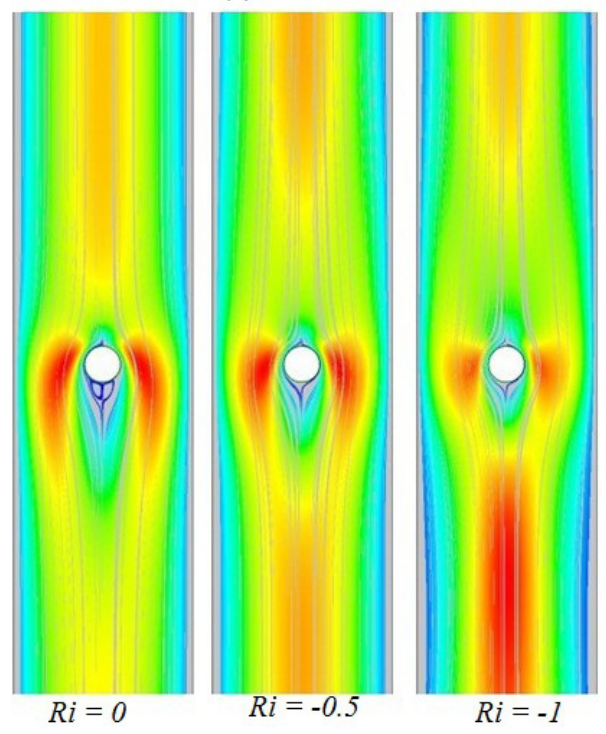

(c) $R e=30$

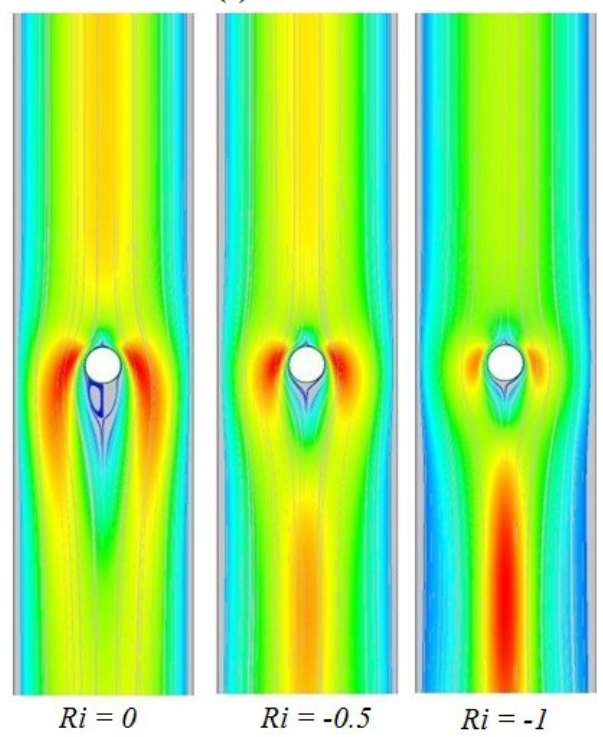

Fig. 4 Streamlines for different $R i$ and $R e$, under the influence of aiding buoyancy. 
This can be attributed by the fact that under assisting buoyancy condition (i.e., when the cylinders are cooled), the velocity of fluid particles behind the cylinder increases and moves towards the steam-wise direction, this inertia force is added with viscous force. Consequently, the recirculation size delays and the point of separation shifts towards the rear stagnation point. On the contrary, for the opposing buoyancy condition (i.e., when the cylinders are heated), behind the cylinder, the inertia force is aided by the buoyancy as well as viscous force. As consequence, the point of separation moves towards the forward stagnation point of the cylinder, causing an early separation as it is observed for the value 10 of Reynolds number. This effect also increases the size of recirculation region for $R e=20$ and 30. Moreover, for the case of opposing buoyancy condition, two symmetrical wake regions are also observed above the cylinder on the channel walls. At fixed Reynolds number, the region size increases with increasing Richardson number. This is due to the effect of opposing thermal buoyancy which increases the velocity of fluid particles around the cylinder toward the upward direction near to channel walls.

The isotherms around the circular cylinder for the range of Reynolds number and Richardson number under opposing and aiding thermal buoyancy are presented in Figs. 5 and 6 respectively. both conditions (opposing and aiding buoyancy), more crowding temperature contours is observed around of the surface of cylinder, which hints the presence of the heat transfer flux between the cylinder surface and downward fluid flow. It is also shown that, the crowding of temperature contours are found to be increased with increasing $R e$ which can be explained by the increase of heat transfer rate with the increase of value of Reynolds number.

Generally, the clustering of isotherms on the front surface is also observed to increase with the gradually increasing value of $R e$ and/or $R i$ (in some cases), it indicates the progressive thinning of the thermal boundary layer which also suggests the heat transfer rate to be the maximum at the front stagnation point. For opposing buoyancy condition, some crowding temperature contours are observed on the channel walls over the obstacle and they increase gradually with $R i$, this behavior reflects the exciting of counter rotating region in these zones. Furthermore, the lateral spread of isotherms is observed to be reduced with increase in the assisting thermal buoyancy (decrease in the value of $R i<0$ ). On other hand, the lateral spread of isotherms is observed to be increased with increase in the opposing thermal buoyancy (increase in the value of $R i>0$ ). (a) $R e=10$
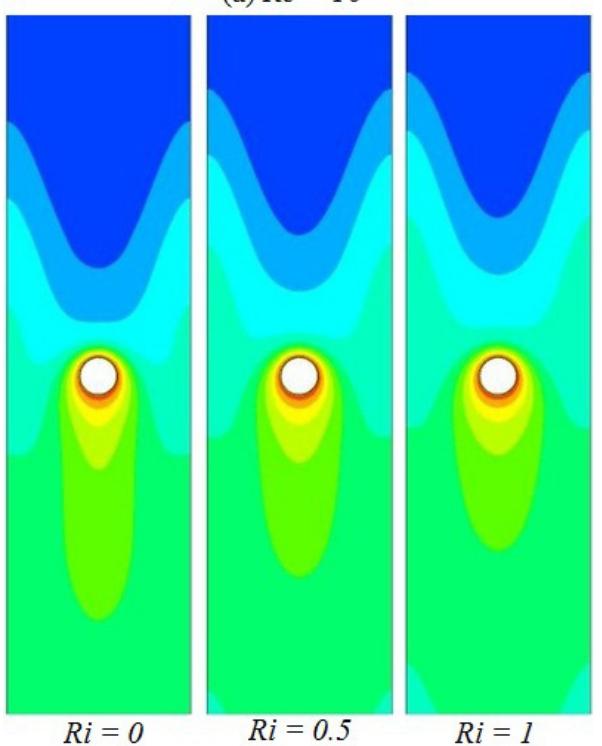

(b) $R e=20$

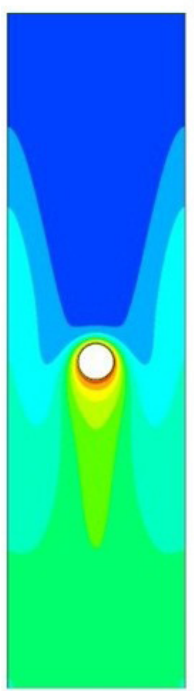

$$
R i=0
$$

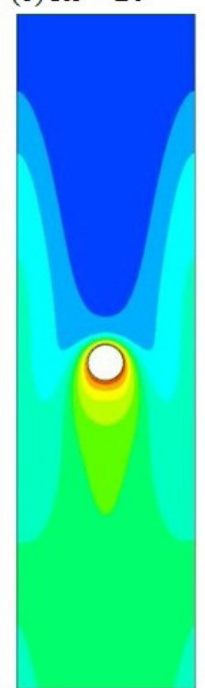

$R i=0.5$

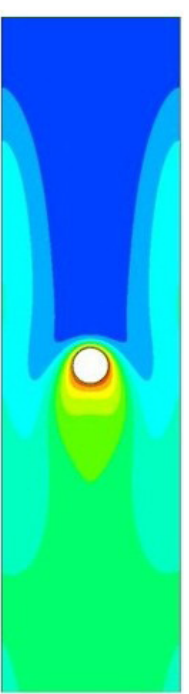

$R i=1$

(c) $R e=30$

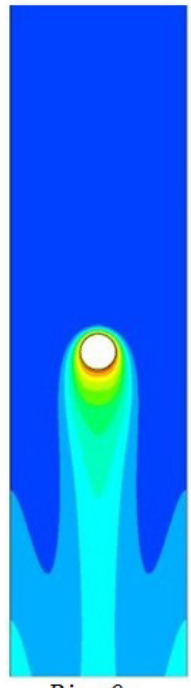

$R i=0$
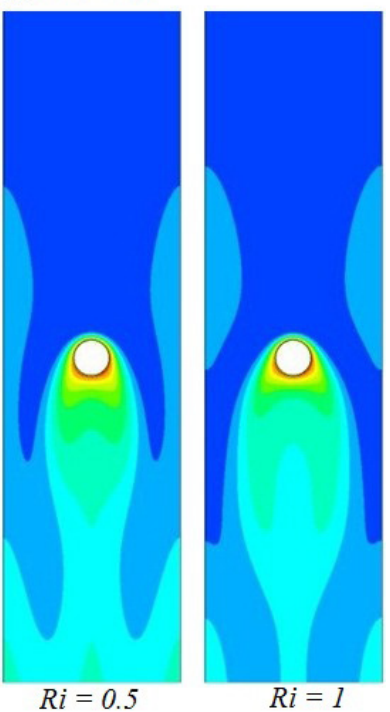

$R i=1$

Fig. 5 Isotherms for different $R i$ and $R e$, under opposing buoyancy. 
(a) $R e=10$

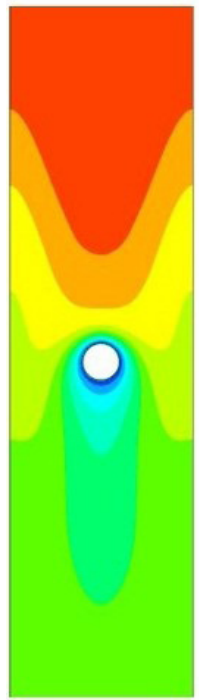

$R i=0$

$R i=-0.5$

(b) $R e=20$

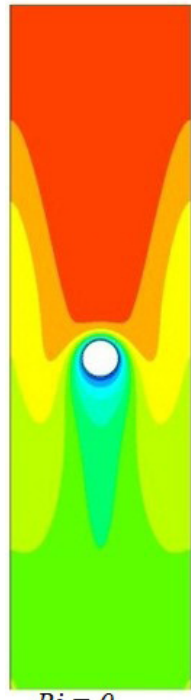

$R i=0$

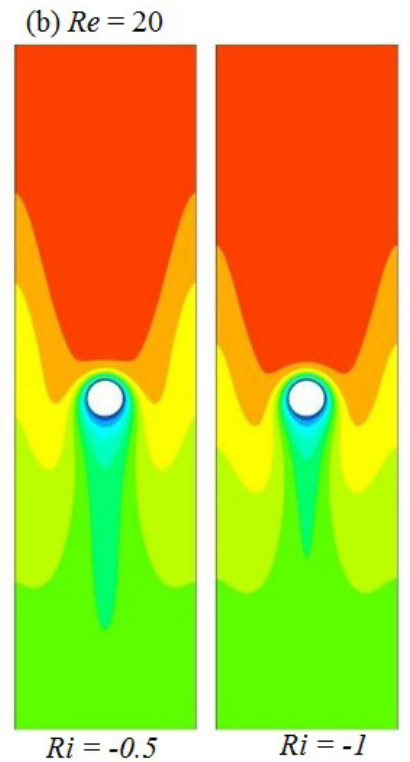

(c) $R e=30$

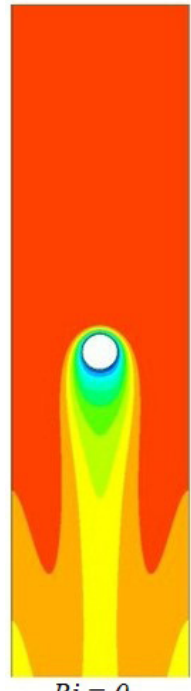

$$
R i=0
$$
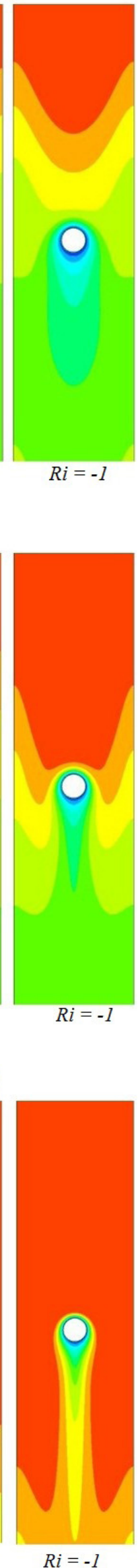

Fig. 6 Isotherms for different $R i$ and $R e$, under aiding buoyancy.

\section{Average Skin Frictional Coefficient}

Fig. 7 and 8 represent the variation of average skin frictional coefficient around the circular cylinder, $C_{f}$, with Richardson number for all values of Reynolds number. The frictional coefficient is defined as:

$$
C_{f}=\frac{\tau_{w}}{\frac{1}{2} \rho v^{2}}
$$

where $\tau_{w}$ is the local wall shear stress, Surface average skin frictional coefficient around the cylinder is obtained by integrating the local wall shear stress along the cylinder face. From these figures, for both buoyancy effects, it is observed that the average frictional coefficient decreases with increase in Reynolds number. For all values of Reynolds number, the friction reduces with increase Richardson number for opposing buoyancy condition, while it increases with decreasing Richardson number for aiding buoyancy condition.

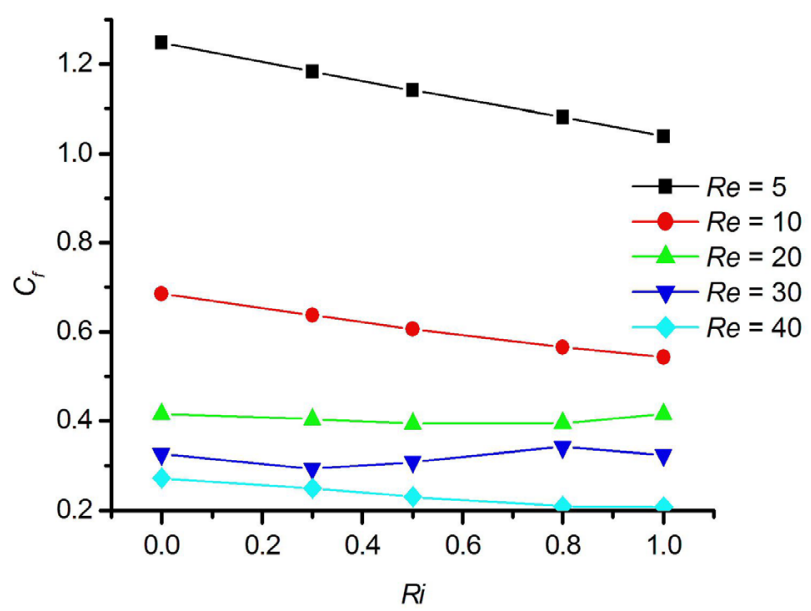

Fig. 7 Variation of average frictional coefficient $C_{f}$ with $R i$ for different value of $R e$, under the influence of opposing buoyancy.

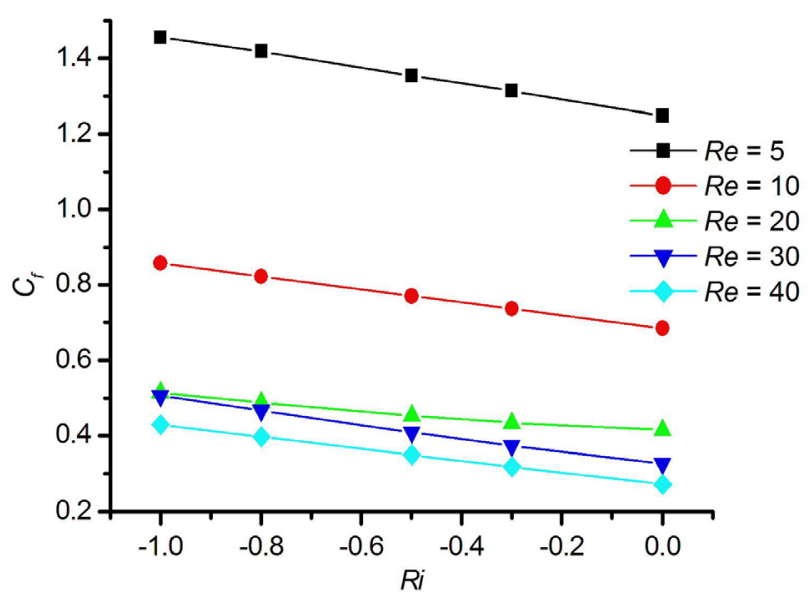

Fig. 8 Variation of average average frictional coefficient $C_{f}$ with $R i$ for different value of $R e$, under the influence of aiding buoyancy. 


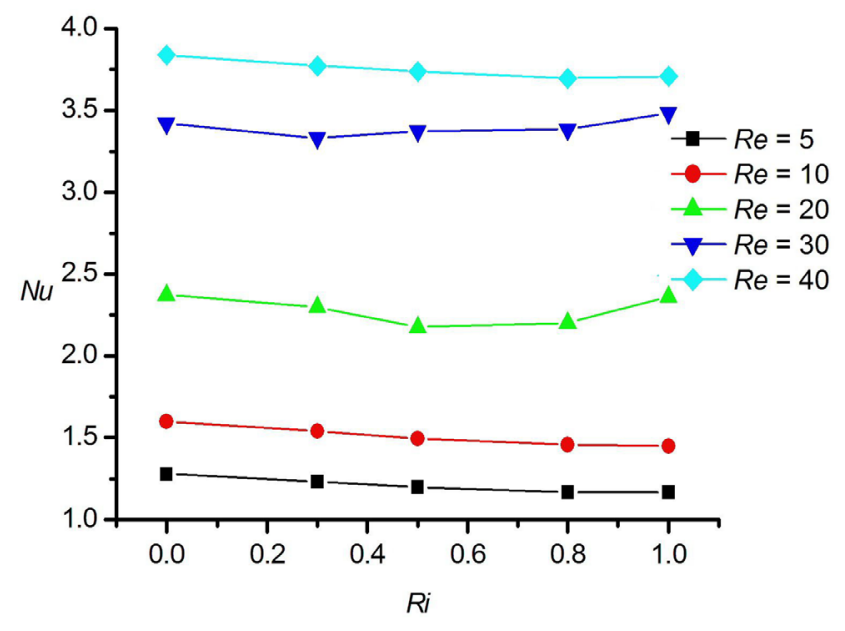

Fig. 9 Variation of average Nusselt number with $R i$ for different value of $R e$, under the influence of opposing buoyancy.

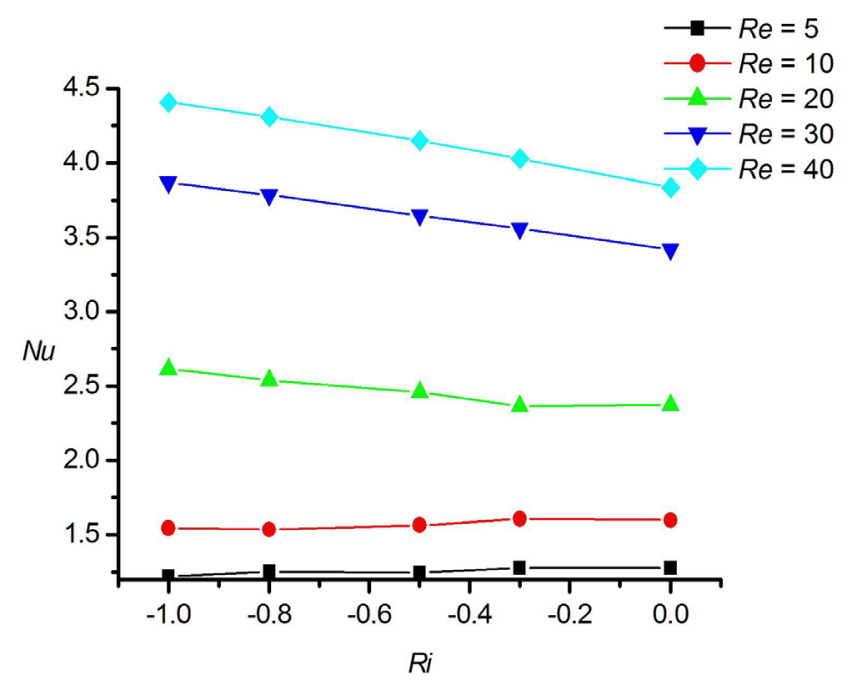

Fig. 10 Variation of average Nusselt number with $R i$ for different value of $R e$, under the influence of aiding buoyancy.

\section{Average Nusset number}

Fig. 9 and 10 depict the variation of surface average Nusselt number on the circular cylinder with Richardson number for different values of Reynolds number under the influence of opposing and aiding thermal buoyancy. For both conditions, it is observed that the average Nusselt number shows positive dependence with Reynolds number due to the gradual thinning of thermal boundary layer with increase in the value of $R e$. interesting variation of Nusselt number is observed with respect to thermal buoyancy condition. For opposing buoyancy, generally increase the effect of opposing buoyancy strength by increasing the Richardson number decreases the heat transfer rate due to the opposite direction of fluid particles in the vicinity of cylinder which decrease the local Reynolds number. On other hand, for the aiding buoyancy condition, increasing the effect of aiding thermal buoyancy force by decreasing $R i$ under the value zero enhances the heat transfer rate due to the increase of velocity of fluid particles towards stream-wise direction which increases the local Reynolds number.

\section{Conclusion}

The numerical simulation is performed to understand the effect of aiding and opposing thermal buoyancy on fluid flow and heat transfer around a vertical circular cylinder for the confined downward flow of Newtonian fluid at low Reynolds number $R e=5$ to 40 , the effect of Reynolds number on the flow and heat transfer is studied for Richardson number range of $R i=-1$ to 1 . It is found that increase in the effect opposing buoyancy increases the instability of the fluid inside the channel and creates an early recirculation zone behind the cylinder. Meanwhile, increase in effect of aiding buoyancy decreases the instability of the fluid and suppresses the recirculation zone behind the cylinder. Moreover, for opposing buoyancy condition, two counter rotating regions are appeared above the cylinder on the channel walls. Generally, increase in the effect of opposing thermal buoyancy reduces frictional coefficient and the heat transfer rate. On other hand, the heat transfer rate and the frictional coefficient are enhanced with increase in the effect of aiding thermal buoyancy.

\section{References}

[1] Laidoudi, H., Bouzit, M. "Suppression of flow separation of power-law fluids flow around a confined circular cylinder by superimposed thermal buoyancy." Mechanika. 23(2), pp. 220-227. 2017. https://doi.org/10.5755/j01.mech.23.2.14342

[2] Salcedo, E., Cajas, C. C., Trevino, C. "Numerical investigation of mixed convection heat transfer from two isothermal circular cylinders in tandem arrangement: buoyancy, spacing ratio, and confinement effects." Theoretical and Computational Fluid Dynamics. 31(2), pp. 159-187. 2017.

https://doi.org/10.1007/s00162-016-0411-z

[3] Chatterjee, D., Mondal, B. "Control of flow separation around bluff obstacles by superimposed thermal buoyancy." Heat and Mass Transfer. 72, pp. 128-138. 2014.

https://doi.org/10.1016/j.ijheatmasstransfer.2014.01.013

[4] Sarkar, S., Dalal, A., Biswas, G. "Mixed convective heat transfer from two identical square cylinders in cross flow at $\mathrm{Re}=100$." Heat and Mass Transfer. 53, pp. 52628-2642. 2011.

https://doi.org/10.1016/j.ijheatmasstransfer.2010.02.053

[5] Chatterjee, D., Raja, M. "Mixed convecton heat transfer past in-line square cylinders in vertical duct." Thermal Science. 17, pp. 567-580. 2013. https://doi.org/10.2298/TSCI101004199C

[6] Sharma, N., Dhiman, A. K., Kumar, S. "Mixed convection flow and heat transfer across a square cylinder under the influence of aiding buoyancy at low Reynolds numbers." Heat and Mass Transfer. 55, pp. 2601-2614. 2012.

https://doi.org/10.1016/j.ijheatmasstransfer.2011.12.034

[7] Sharma, A., Eswaran, V. "Effect of channel confinement and aiding/opposing buoyancy on the two-dimensional laminar flow and heat transfer across a square cylinder." Heat and Mass Transfer, 48, pp. 5310-5322. 2005 .

https://doi.org/10.1016/j.ijheatmasstransfer.2005.07.027 
[8] Salcedo, E. , Trevino, C., Cajas, J. C., Martínez-Suástegui, L. "Unsteady mixed convection from two isothermal semicircular cylinders in tandem arrangement." In: Sohel Murshed, S. M. (Ed.) Heat Exchangers - Design, Experiment and Simulation. InTech, 2017. https://doi.org/10.57772/66692

[9] Gupta, S. K. , Ray, S. Chatterjee, D. "Steady Mixed Convection in Power-Law Fluids from a Heated Triangular Cylinder." Heat Transfer Engineering. 2017.

https://doi.org/10.1080/01457632.2017.1357773

[10] Singh, G., Makinde, O. D. "Axisymmetric Slip Flow on a Vertical Cylinder with Heat Transfer." Sains Malaysiana. 43(3), pp. 483-489. 2014. https://doi.org/10.57772/66692

[11] Yahiaoui, K., Nehari, D., Draoui, B. "The Investigation of the Mixed Convection from a Confined Rotating Circular Cylinder." Periodica Polytechnica Mechanical Engineering. 61(3), pp. 161-172. 2017. https://doi.org/10.3311/PPme.9338
[12] Chatterjee D., Amiroudine, S. "Two-dimensional mixed convection heat transfer from confined tandem square cylinders in cross-flow at low Reynolds numbers." International Communications in Heat and Mass Transfer. 37, pp. 7-16. 2010.

https://doi.org/10.1016/j.icheatmasstransfer.2009.10.007

[13] Sukesan, M. K. , Dhiman, A. K. "Laminar mixed convection in a channel with a built-in semi-circularcylinder under the effect of cross-buoyancy." Communication in Heat and Mass Transfer. 58, pp. 25-32. 2014. https://doi.org/10.1016/j.icheatmasstransfer.2014.08.025

[14] Laidoudi, H., Bouzit, M. "The effect of asymmetrically confined circular cylinder and opposing buoyancy on fluid flow and heat transfer." Defect and Diffusion Forum. 374, pp. 18-28. 2017.

https://doi.org/10.4028/www.scientific.net/DDF.374.18

[15] ANSYS Workbench User's Guide, Release 13.0, ANSYS Inc., Canonsburg, PA, 2010. 